\title{
Morphology-controlled synthesis of Ni-MOFs with highly enhanced electrocatalytic performance for urea oxidation
}

Mengwei Yuan, ${ }^{\dagger}$ Rui Wang, ${ }^{\dagger}$ Zemin Sun, Liu Lin, Han Yang, Huifeng Li, Caiyun Nan, Genban Sun,* and Shulan Ma*

Beijing Key Laboratory of Energy Conversion and Storage Materials and College of Chemistry, Beijing Normal University, Beijing 100875, China 
Table S1 The composition of the as-prepared Ni-MOF-100

\begin{tabular}{lllll}
\hline Ni-MOF-100 & $\mathrm{Ni}\left(\mathrm{N}_{2} \mathrm{C}_{4} \mathrm{H}_{5}\right)_{2.068}\left(\mathrm{H}_{2} \mathrm{O}\right)_{0.239}\left(\mathrm{NO}_{3}\right)_{0.034}$ & $\mathrm{M}=232.6 \mathrm{~g} / \mathrm{mol}$ \\
\hline Ni-MOF-100 & $\mathrm{Ni}(\mathrm{wt} \%)$ & $\mathrm{C}(\mathrm{wt} \%)$ & $\mathrm{N}(\mathrm{wt} \%)$ & $\mathrm{H}(\mathrm{wt} \%)$ \\
Mea. & 25.35 & 42.90 & 25.23 & 4.636 \\
Cal. & 25.23 & 42.67 & 25.10 & 4.651 \\
\hline
\end{tabular}


Table S2 The fitting values of EIS parameters of Ni-MOFs catalysts for urea electro-oxidation in $\mathrm{KOH}$ system.

\begin{tabular}{ccccccc}
\hline Sample & $\mathrm{R}_{\mathrm{s}}$ & $\mathrm{R}_{1}$ & $\mathrm{CPE}_{1}$ & $\mathrm{R}_{2}$ & $\mathrm{CPE}_{2}$ & $\mathrm{X}^{2}$ \\
\hline Ni-MOF-100 & 10.67 & 1.147 & $4.972 \mathrm{u}$ & 5.330 & $40.89 \mathrm{u}$ & $4.4 \mathrm{E}-3$ \\
Ni-MOF-80 & 9.927 & 1.815 & $5.471 \mathrm{u}$ & 9.164 & $28.53 \mathrm{u}$ & $7.5 \mathrm{E}-3$ \\
Ni-MOF-60 & 10.76 & 3.683 & $6.980 \mathrm{u}$ & 9.720 & $29.94 \mathrm{u}$ & $4.5 \mathrm{E}-3$ \\
Ni-MOF-RT & 16.64 & 81.05 & $2.535 \mathrm{u}$ & 206.9 & $11.42 \mathrm{u}$ & 3.3 \\
\hline
\end{tabular}

Note: The units: R (ohm $\left.\mathrm{cm}^{2}\right)$; CPE (F). 
Table S3 Comparison of the Ni-based electrocatalysts for UOR

\begin{tabular}{|c|c|c|c|}
\hline Sample & $\begin{array}{l}\text { Potential @10 mA } \\
\mathrm{cm}^{-2} \text { (V vs. RHE) }\end{array}$ & Substrate & Reference \\
\hline Ni-MOF nanowires & 1.36 & Glassy carbon & This work \\
\hline Metallic $\mathrm{Ni}(\mathrm{OH})_{2}$ & 1.39 & Glassy carbon & Angew. Chem. Int. Ed., 2016 ${ }^{\mathrm{S} 1}$ \\
\hline $\mathrm{Ni} / \mathrm{Pd}-\mathrm{C}$ fiber & 1.51 & Glassy carbon & Applied Surface Science, $2018^{\mathrm{S} 2}$ \\
\hline $\mathrm{Ni}-\mathrm{Cr}$ & 1.38 & Glassy carbon & ChemCatChem $2017^{\mathrm{S3}}$ \\
\hline $\mathrm{LaNiO}_{3}$ & 1.39 & Glassy carbon & ACS Catal., 2016 ${ }^{\mathrm{S} 4}$ \\
\hline $\mathrm{Ni}-\mathrm{Co}$ & 1.53 & Glassy carbon & Sci. Rep., $\mathbf{2 0 1 4}^{\mathrm{S5}}$ \\
\hline $\mathrm{Ni} / \mathrm{N}, \mathrm{S}-\mathrm{CNFs}$ & $\sim 1.40$ & Glassy carbon & J. Colloid Interface Sci., $2018^{\mathrm{S} 6}$ \\
\hline Graphene-Ni(OH $)_{2}$ & 1.52 & Glassy carbon & Electrochim. Acta, $\mathbf{2 0 1 3}^{\mathrm{S} 7}$ \\
\hline $\mathrm{Ni} / \mathrm{C}$ & $\sim 1.38$ & Glassy carbon & ACS Appl. Mater. Interfaces $2018^{\mathrm{S} 8}$ \\
\hline
\end{tabular}

S1. Zhu, X.; Dou, X.; Dai, J.; An, X.; Guo, Y.; Zhang, L.; Tao, S.; Zhao, J.; Chu, W.; Zeng, X. C.; Wu, C.; Xie, Y. Metallic Nickel Hydroxide Nanosheets Give Superior Electrocatalytic Oxidation of Urea for Fuel Cells. Angew. Chem. Int. Ed. 2016, 55, 12465-12469.

S2. Mohamed, I. M. A.; Yasin, A. S.; Barakat, N. A. M.; Song, S. A.; Lee, H. E.; Kim, S. S. Electrocatalytic behavior of a nanocomposite of Ni/Pd supported by carbonized PVA nanofibers towards formic acid, ethanol and urea oxidation: A physicochemical and electro-analysis study. Appl. Surf. Sci. 2018, 435, 122-129.

S3. Singh, R. K.; Schechter, A. Electroactivity of NiCr Catalysts for Urea Oxidation in Alkaline Electrolyte. Chemcatchem 2017, 9, 3374-3379.

S4. Forslund, R. P.; Mefford, J. T.; Hardin, W. G.; Alexander, C. T.; Johnston, K. P.; Stevenson, K. J. Nanostructured $\mathrm{LaNiO}_{3}$ Perovskite Electrocatalyst for Enhanced Urea Oxidation. ACS Catal. 2016, 6, 5044-5051.

S5. Xu, W.; Zhang, H.; Li, G.; Wu, Z. Nickel-cobalt bimetallic anode catalysts for direct urea fuel cell. Sci. Rep. 2014, 4, 5863.

S6. Liu, D.; Li, W.; Li, L.; Ling, H.; You, T. Facile preparation of Ni nanowire embedded nitrogen and sulfur dual-doped carbon nanofibers and its superior catalytic activity toward urea oxidation. $J$. Colloid Interface Sci. 2018, 529, 337-344.

S7. Wang, D.; Yan, W.; Vijapur, S. H.; Botte, G. G. Electrochemically reduced graphene oxidenickel nanocomposites for urea electrolysis. Electrochim. Acta 2013, 89, 732-736.

S8. Wang, L.; Ren, L.; Wang, X.; Feng, X.; Zhou, J.; Wang, B. Multivariate MOF-Templated Pomegranate-Like Ni/C as Efficient Bifunctional Electrocatalyst for Hydrogen Evolution and Urea Oxidation. ACS Appl. Mater. Interfaces 2018, 10, 4750-4756. 


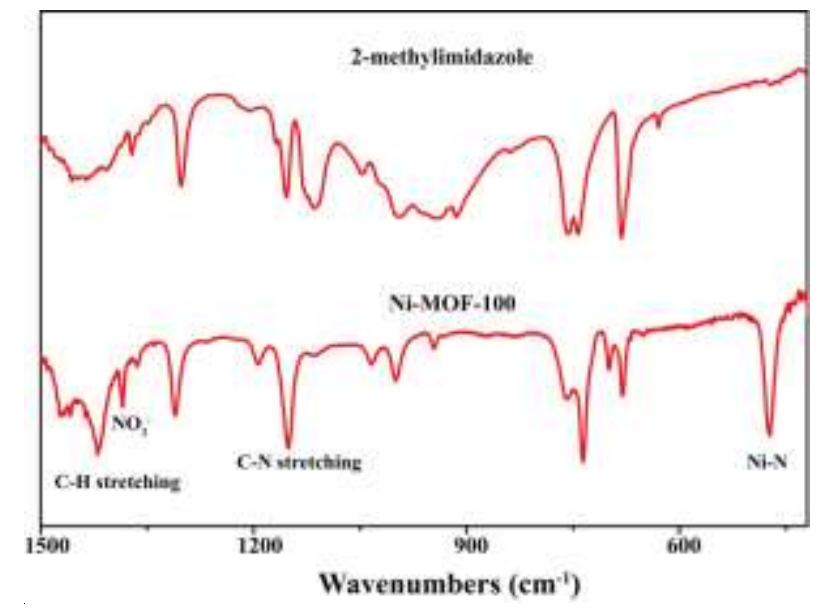

Figure S1 The FT-IR spectra of 2-methylimidazole and Ni-MOF-100. 


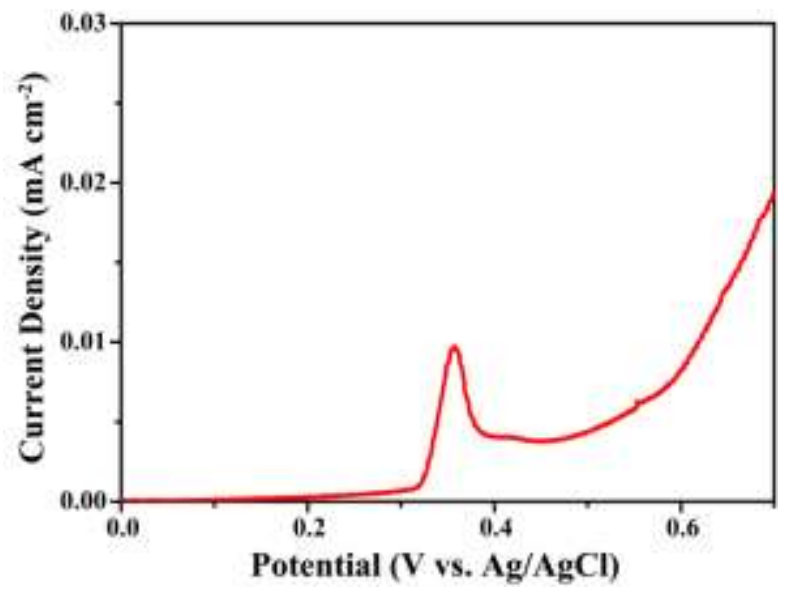

Figure S2 The LSV curve of Ni-MOF-60 in $1.0 \mathrm{M} \mathrm{KOH}$. 


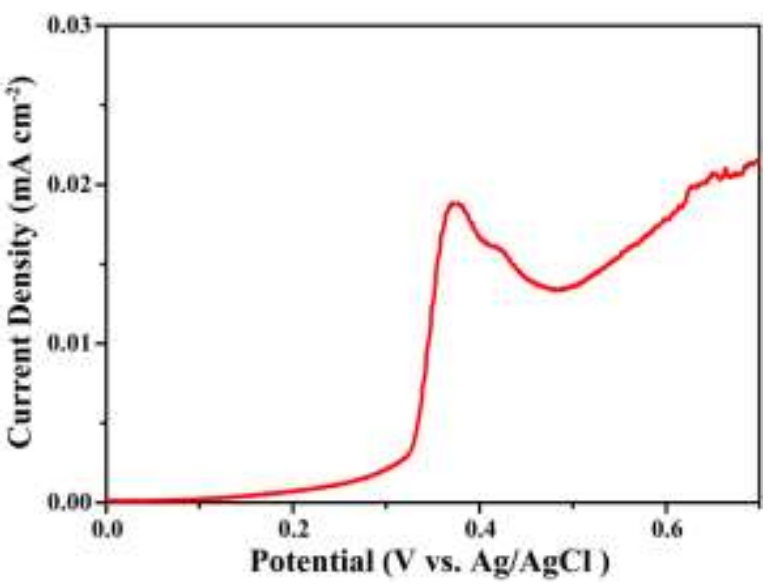

Figure S3 The LSV curve of Ni-MOF-80 in $1.0 \mathrm{M} \mathrm{KOH}$. 

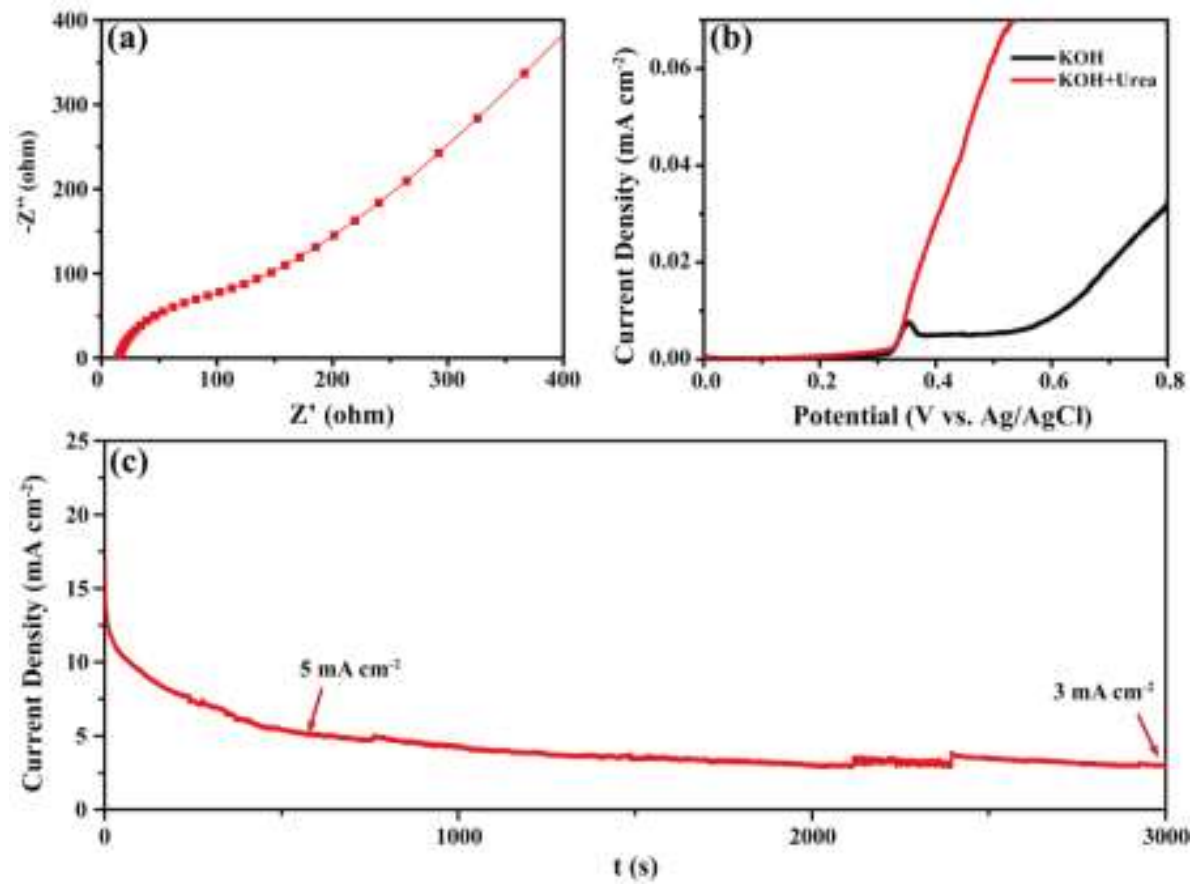

Figure S4 (a) EIS, (b) LSV Curves and (c) i-t measurement of Ni-MOF-RT. 